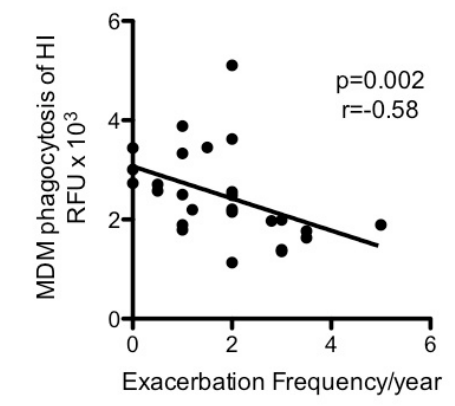

Figure 1. Relationship between MDM phagocytosis of fluorescentlylabelled Haemophilus influenzae and exacerbation frequency.

Abstract P253 Figure 1

\section{P254 IDENTIFYING MMP-12 SUBSTRATES AS THERAPEUTIC TARGETS IN COPD}

doi:10.1136/thoraxjnl-2012-202678.346

'B Mallia-Milanes, 'D Clements, ${ }^{2} \mathrm{~A}$ Sheehan, 'C Bolton, 'SR Johnson. 'University of Nottingham, Nottingham, UK; ${ }^{2}$ Nottingham University Hospitals NHS Trust, Nottingham, UK

Background Matrix metalloprotease (MMP)-12 is a key protease in COPD which cleaves pulmonary extracellular matrix and nonmatrix substrates. Variation in MMP-12 activity affects severity of COPD, yet the mechanism of this, including MMP-12's non-matrix substrates in COPD lungs are unknown. Targeting MMP-12 substrates may lead to the development of drugs for COPD with reduced side effects compared to the broader spectrum MMP inhibitors.

Aims To identify MMP-12 substrates of relevance to COPD and determine how their activity affects disease progression in vitro and in vivo.

Methods In vitro cleavage assays: After literature review the proinflammatory mediators osteopontin and tumour necrosis factor (TNF)- $\alpha$ were selected as potential MMP-12 substrates in COPD. Both were incubated with MMP-12 and reaction products analysed by silver stain and western blot. EDTA was used as a metalloprotease inhibitor and thrombin as positive control. COPD cohort: Patients with COPD were recruited during exacerbations at the Nottingham University Hospitals NHS Trust. Sputum, lung function and other data were collected on Day 0 and 1 and 4 weeks later. Sputum was analysed by western blot for proteins of interest. The study was approved by the local research ethics committee and all patients gave informed consent.

Results MMP-12 cleaved osteopontin and pro-TNF- $\alpha$ in a dose and time-dependent manner when visualised by silver staining. Cleavage was dependent on MMP-12 activity as it was inhibited by EDTA. Western blot of cleaved protein fragments gave a characteristic band signature. MMP-12 was present in sputum of patients with COPD as demonstrated by western blotting, ELISA and casein zymography. Western blot analysis of sputum with anti-osteopontin antibodies showed a similar band signature to the in vitro cleavage suggesting osteopontin is cleaved in the airways of patients with COPD.

Discussion MMP-12 possesses proteolytic activity against osteopontin and pro-TNF- $\alpha$ in vitro. MMP-12, osteopontin and TNF $\alpha$ are present in COPD sputum and our data suggest that MMP-12 may target osteopontin in COPD. Further work is needed to determine the precise mechanisms of such MMP-12 substrate activity in COPD.

\section{P255 THE EFFECTS OF HYPOXIA ON NEUTROPHIL DEGRANULATION}

doi:10.1136/thoraxjnl-2012-202678.347

K Hoenderdos, L Porter, S Alam, N Mc Govern, C Fiddler, J Skepper, ER Chilvers, AM Condliffe. University of Cambridge, Cambridge, Cambridgeshire

Tissues such as the skin and intestinal epithelium experience physiological hypoxia whereas pathological hypoxia occurs at inflammatory sites. Neutrophils are recruited to infective/inflamed areas and are thus required to operate under low oxygen tensions. We have shown previously that hypoxia delays neutrophil apoptosis (JEM 2005; 201:105) and impairs bacterial killing (J Immunol 2011; 186:453) and have now studied the effect of hypoxia on the release of histotoxic neutrophil proteases.

Neutrophils isolated from healthy volunteers were subjected to normoxia or hypoxia $(3 \mathrm{kPa})$. Superoxide anion release was measured by the reduction of cytochrome. C Elastase release was quantified by the cleavage of labelled elastin.

Hypoxic incubation for 4 hours resulted in a 3-fold reduction in superoxide anion release from cells stimulated with GM-CSF and fMLP. In contrast, elastase release from the azurophilic granules was augmented almost 3-fold under hypoxia. The release of MMP-9 and lactoferrin was similarly up-regulated, suggesting a more generalised increase in degranulation under hypoxia. In addition to this electron microscopy showed that hypoxia induced a more activated phenotype (e.g. increased membrane ruffling and cell spreading).

We show that hypoxia can induce a more destructive neutrophil phenotype, with enhanced degranulation and release of potentially histotoxic proteases, impaired bacterial killing, and delayed apoptosis. These data suggest that hypoxia aversively affects neutrophil function and may augment neutrophil mediated tissue destruction.

\section{P256 P2X4 AND NNOS EXPRESSION IN HUMAN CILIATED AIRWAY EPITHELIUM}

doi:10.1136/thoraxjnl-2012-202678.348

I Premadeva, HL Lenartowicz, J Underwood, PM Lackie, JS Lucas, CL Jackson. University of Southampton, Faculty of Medicine, Southampton, UK

Background Calcium $\left(\mathrm{Ca}^{2+}\right)$ and nitric oxide (NO) modulate ciliary beat frequency (CBF). NO synthase (NOS) isoenzymes, responsible for NO production, localise in human airway epithelium and constitutive NOS activity is dependant on $\mathrm{Ca}^{2+}$. Adenosine triphosphate gated purinergic ion channels, comprised of $\mathrm{P}_{2} \mathrm{X}_{1-7}$ subunits, govern $\mathrm{Ca}^{2+}$ influx and $\mathrm{P} 2 \mathrm{X}_{4}$ reportedly localises to rabbit airway cilia. Also, P2X proteins co-localise with neuronal NOS (nNOS) in guinea pig cochlea outer hair cells and rat hypothalamus. We hypothesised that $\mathrm{P}_{2} \mathrm{X}_{4}$ and $\mathrm{nNOS}$ co-localise in human airway cilia and are involved in modulating CBF.

Objectives To determine $P 2 X_{1,3-7}$ mRNA expression and $\mathrm{P} 2 \mathrm{X}_{4}$ and nNOS localisation in human nasal epithelium, and assess their possible interaction in CBF modulation.

Methods and Results Relative to $\beta$-actin expression, consistent and moderate $P 2 X_{4,6}$ mRNA and variable $P 2 X_{3,5,7}$ mRNA were detected by RT-PCR in $(n=4)$ human primary airway epithelium cultured at air-liquid interface (ALI). In ALI cultured primary epithelium $\mathrm{P}_{2} \mathrm{X}_{4}$ localised $(\mathrm{n}=3)$ to cell membranes and cytoplasm and $\mathrm{nNOS}$ localised to cilia $(\mathrm{n}=3)$ by immunofluorescence. P2X localised to the ciliary tips $(n=2)$, whilst nNOS localised to the proximal portion of cilia (specificity confirmed by blocking peptide) in nasal polyp paraffin wax sections by immunohistochemistry $(n=6)$. High speed video microscopy confirmed a 30 minute baseline $\mathrm{CBF}$ at $37^{\circ} \mathrm{C}(12.9 \mathrm{~Hz} \mathrm{SD} \pm 0.8, \mathrm{n}=5)$ on nasal epithelium biopsies 